\title{
MATHEMATICS
}

\section{ON THE MEMBERSHIP QUESTION IN SOME LINDENMAYER-SYSTEMS}

\author{
BY
}

P. G. DOUCET

(Communicated by Prof. A. van Wijngandoen at the meeting of June 26, 1971)

\section{Summary}

Lindenmayer-systems are a family of string-generating systems, and several types can be distinguished, of different strength. The subject of this article is the membership question in the languages generated by determinate, no-input Lindenmayer-systems (or, shortly, D0L-languages). For propagating D0L-systems (which contain no production rules yielding the empty string) the question is easily answered. For D0L-systems in general it can be solved in two steps, involving the construction of an intermediate "backbone" system, which is propagating.

\section{NOTATION; DESCRIPTION OF DOL-SYSTEMS}

The notation conforms to common usage in language theory, and I shall only state a few conventions.

Any sequence of elements of an alphabet $\Sigma$ is called a word over $\Sigma$; $\Lambda$ is the empty word.

If $\sigma \in \Sigma$, then $\sigma^{2}=\sigma \sigma$, etc.; $\sigma^{0}=\Lambda$.

$\Sigma^{*}$ is the Kleenean closure of $\Sigma$.

$\# \Sigma$ denotes the number of elements of $\Sigma$.

$|w|$ denotes the length of a word $w$.

If $\delta$ is a mapping of a set into itself, then $\delta^{2}(x)=\delta(\delta(x))$, etc.; $\delta^{0}(x)=x$. Elements (or letters) of an alphabet shall usually be denoted by $\sigma, \tau, \ldots$, words by $u, v, w, x, \ldots$.

$L$-systems were first proposed by A. LINDENMAYER in 1968 [3], as a model for the development of filamentous organisms. D0L-systems, which form a subclass of the L-systems, can be formally described as follows:

Defrnimion. A $D O L$-system is an ordered triple $G=\left\langle\Sigma, \delta, w_{0}\right\rangle$, where $\Sigma$ (the alphabet) is a finite, nonempty set, $\delta$ (the set of productions) is a total mapping from $\Sigma$ into $\Sigma^{*}$, and $w_{0}$ (the axiom) is a non-empty word over $\Sigma$.

The domain of $\delta$ is extended from $\Sigma$ to $\Sigma^{*}$ in a natural way, by defining 1. $\delta(\Lambda)=\Lambda$.

2. $\delta(\sigma w)=\delta(\sigma) \delta(w)$ for all $\sigma \in \Sigma$ and $w \in \Sigma^{*}$. 
The relation $\Rightarrow$ in DoL-systems (and in L-systems in general) is not the same as in the usual grammars:

Definition. Let $G=\left\langle\Sigma, \delta, w_{0}\right\rangle$ be a DoL-system.

1. $w_{1} \Rightarrow w_{2}$ if and only if $\delta\left(w_{1}\right)=w_{2}$.

2. $\quad w_{1} \stackrel{*}{\Rightarrow} w_{2}$ if and only if $\delta^{m}\left(w_{1}\right)=w_{2}$ for some $m \geqslant 0$.

3. The language of $G$ is defined by $L(G)=\left\{w: w_{0} \stackrel{*}{\Rightarrow} w\right\}$.

Note that

(a) if $w_{1} \Rightarrow w_{2}$, then $w_{2}$ is the result of a $\delta$-mapping applied to each letter of $w_{1}$.

(b) there is no distinction between terminal and non-terminal letters, and every word derived from $w_{0}$ is in $L(G)$.

These properties are characteristic for L-systems; they account for many of the differences with other grammars.

If $\delta(\sigma) \neq \Lambda$ for all $\sigma \in \Sigma$, then $G$ is called a propagating D0L-system (or, with biological connotations, without cell-death), abbreviated as PD0L-system.

Example 1. $G=\langle\{0,1\}, \delta, 011\rangle$ with $\delta$ as follows:

$0 \rightarrow 1001,1 \rightarrow \Lambda$.

From 011 , words are produced as follows:

$011 \Rightarrow 1001 \Rightarrow 10011001 \Rightarrow(1001)^{4} \Rightarrow(1001)^{8}$ etc.

So $L(G)=\{011\} \cup\left\{(1001)^{2 n}: n \geqslant 0\right\}$.

$G$ is a DoL-system; it is not propagating.

EXAMPLE 2. $H=\langle\{0,1\}, \delta, 10\rangle$, with $\delta: 0 \rightarrow 00,1 \rightarrow 0,1 \rightarrow 1$.

$H$ is propagating; but it is no D0L-system, the two-valuedness of $\delta(1)$ violating the condition that $\delta$ be a mapping from $\Sigma$ into $\Sigma^{*}$.

\section{PD0L-SYSTEMS}

Lemma 1. Let $G=\left\langle\Sigma, \delta, w_{0}\right\rangle$ be a PD0L-system.

If $L(G)$ is infinite, then it contains at most \# $\Sigma$ words of a given length; formally:

$$
(\forall k)(\#\{w \in L(G):|w|=k\} \leqslant \# \Sigma)
$$

Proof: Because $L(G)$ is infinite, $\delta$ contains some strictly increasing production rules (i.e., with $|\delta(\sigma)| \geqslant 2$ ).

Because $G$ is propagating, word length cannot decrease, so all words of the same length follow immediately upon each other.

Let $w_{1}$ and $w_{z}$ be the first and last words of length $k$. Then $w_{z}$ contains at least one letter, say $\sigma_{z}$, with $\left|\delta\left(\sigma_{z}\right)\right| \geqslant 2$.

Let $\sigma_{1} \Rightarrow \sigma_{2} \Rightarrow \ldots \Rightarrow \sigma_{z-1} \Rightarrow \sigma_{z}$ (with each $\sigma_{i}$ in $w_{i}$ ). Then $\left|\delta\left(\sigma_{i}\right)\right|=1$ for all $\sigma_{i}$ except $\sigma_{z}$.

Now all $\sigma_{1}, \ldots, \sigma_{z}$ have to be difforent (for, if two of them were equal, $\sigma_{z}$ would also be equal to some $\sigma_{y}$; but $\left|\delta\left(\sigma_{y}\right)\right|=1$ while $\left|\delta\left(\sigma_{z}\right)\right| \geqslant 2$, and in a determinate system this contradicts $\sigma_{\nu}=\sigma_{z}$ ), and this implies $z \leqslant \# \Sigma$. 
Corollary: In PD0L-systems the membership question is effectively solvable.

The decision procedure is to simply start producing words from $w_{0}$ on, and stop as soon as either some previous word reappears (in which case $L(G)$ is finite), or a word longer than $w$ (the word in question) is produced. Lemma 1 assures us that, if $L(G)$ is infinite, this will happen in at most $\left(|w|+1-\left|w_{0}\right|\right) \cdot \not \Sigma$ production steps.

Lemma 2. Let $G=\left\langle\Sigma, \delta, w_{0}\right\rangle$ be a DoL-system. If there exist different $m_{1}$ and $m_{2}$ such that $\delta^{m_{1}}\left(w_{0}\right)=\delta^{m_{2}}\left(w_{0}\right)$, then $L(G)$ is finite.

Proof: Let $m_{2}=m_{1}+q$ with $q>0$.

For any $z>m_{2}, z=m_{1}+\lambda q+\mu$ for some $\lambda \geqslant 1$ and $0 \leqslant \mu<q$.

Then $\delta^{z}\left(w_{0}\right)=\delta^{m_{1}+\lambda a+\mu}\left(w_{0}\right)=\delta^{m_{1}+\mu}\left(w_{0}\right)$.

Consequently, no new words are produced after $w_{m_{2}}$. So $L(G)$ is finite.

The converse of lemma 2 also holds.

Lemma 3. Let $G=\left\langle\Sigma, \delta, w_{0}\right\rangle$ be a PD0L-system with \# $\Sigma=n$.

1. It is effectively decidable whether $L(G)$ is finite.

2. If $L(G)$ is finite, then $\# L(G) \leqslant n^{n}+n$.

Proof: I shall use the following conventions:

- $\sigma \in \Sigma$ will be called ultimately periodic if

$$
(H a \geqslant 0, \pi \geqslant 1)\left(\delta^{a+\pi}(\sigma)=\delta^{a}(\sigma)\right) .
$$

Note that the smallest among possible $a$ 's is always smaller than $n$. The smallest among different $\pi$ 's is called the period of $\sigma$. Note that this period is never larger than $n$.

$-\Sigma$ can be partitioned as follows:

$$
\begin{aligned}
& \Sigma_{1}=\left\{\sigma \in \Sigma:\left|\delta^{n}(\sigma)\right|=1\right\} . \\
& \Sigma_{2}=\Sigma-\Sigma_{1} .
\end{aligned}
$$

1. Obviously,

and

$$
\text { if } \sigma \in \Sigma_{1} \text {, then }\left|\delta^{p}(\sigma)\right|=1 \text { for all } p
$$

$$
\text { if } \sigma \in \Sigma_{1} \text {, then } \sigma \text { is ultimately periodic. }
$$

One can determine $\delta^{n}(\sigma)$ for each $\sigma \in \Sigma$, and then see whether or not $\delta^{n}(\sigma) \in \Sigma_{1}^{*}$.

a. If $\delta^{n}(\sigma) \in \Sigma_{1}{ }^{*}$, then $\sigma$ is ultimately periodic; for, if $\delta^{n}(\sigma)=\sigma_{1} \ldots \sigma_{m}$ and each $\sigma_{i}$ is ultimately periodic with period $\pi_{i}$, then

$$
\delta^{n+\pi_{1} \cdot \pi_{2} \ldots \pi_{m}}(\sigma)=\delta^{n}(\sigma) \text {. }
$$

b. If $\delta^{n}(\sigma) \notin \Sigma_{1}{ }^{*}$, then $\delta^{n}(\sigma)=\ldots \tau_{n} \ldots$ for some $\tau_{n} \in \Sigma_{2}$.

Consider $\tau_{n}$ with its ancestors: $\tau_{0}(=\sigma), \tau_{1}, \ldots, \tau_{n-1}, \tau_{n}$.

According to (i), $\tau_{i} \in \Sigma_{2}$ for each $\tau_{i}$. 
As $\# \Sigma_{2} \leqslant \# \Sigma=n$, at least two of the $\tau_{i}$ 's must be equal, say, $\tau_{k}$ and $\tau_{k+j}$.

Then $\delta^{j}\left(\tau_{k}\right)=w_{1} \tau_{k+j} w_{2}=w_{1} \tau_{k} w_{2}$.

$\tau_{k} \in \Sigma_{2}$, so $w_{1}$ and $w_{2}$ cannot both be empty. If one considers $\delta^{2 j}\left(\tau_{k}\right)$, $\delta^{3 j}\left(\tau_{k}\right)$ etc., one observes that the offspring of $\tau_{k}$ (and of $\sigma$ ) strictly increases in length. So $\sigma$ is not ultimately periodic.

The above argument shows that, for every $\sigma \in \Sigma$, it can be determined whether $\sigma$ is ultimately periodic. Now, if all letters in $w_{0}$ are ultimately periodic, the same holds for $w_{0}$ itself; and then, by lemma $2, L(G)$ is finite. If, on the other hand, not all letters in $w_{0}$ are ultimately periodic, then obviously $L(G)$ is infinite.

2. Consider some $\sigma_{i}$ in $w_{0}$. Since $L(G)$ is finite, $\sigma_{i}$ is ultimately periodic. As shown in $1^{a}$ (and in the same notation),

$$
\delta^{n+\pi_{1} \pi_{2} \ldots \pi_{m}}\left(\sigma_{i}\right)=\delta^{n}\left(\sigma_{i}\right)
$$

By simple concatenation one obtains a similar relation for $w_{0}$ instead of $\sigma_{t}$. Taking into account that only different $\pi_{i}$ 's have to be included in the left-hand exponent, each of them no larger than $n$, it is clear that this exponent can always be made smaller than $n^{n}+n$. This sets an upper bound to $\# L(G)$.

\section{Propagating sub-systems of D0l-systems}

From any D0L-system $G$ a simpler system $\tilde{G}$ can be constructed, which is propagating. The construction is as follows:

Let $G=\left\langle\Sigma, \delta, w_{0}\right\rangle$ be a D0L-system with \# $\#=n$.

Define an erasing homomorphism $\chi$ on $\Sigma \cup\{\Lambda\}$ :

$$
\begin{array}{r}
\chi(\sigma)=\sigma \text { if } \delta^{n}(\sigma) \neq \Lambda \\
A \text { if } \delta^{n}(\sigma)=\Lambda .
\end{array}
$$

$\chi$ is extended to words over $\Sigma^{*}$ by

Now define

$$
\chi(\sigma * w)=\chi(\sigma) * \chi(w)
$$

$$
\begin{aligned}
& \Sigma=\{\sigma \in \Sigma: \chi(\sigma)=\sigma\} \\
& \tilde{\delta}=\chi \circ \delta, \text { defined on } \tilde{\Sigma} \\
& \tilde{w}=\chi(w) \text { for every } w \in \Sigma^{*} \\
& \tilde{G}=\langle\tilde{\Sigma}, \tilde{\delta}, \tilde{w}\rangle .
\end{aligned}
$$

The following example illustrates the construction of $\widetilde{G}$ from $G$.

Let $G=\langle\{1,2,3,4\}, \delta, 44231\rangle$ with

$$
\delta(1)=42 \quad \delta(2)=3 \quad \delta(3)=\Lambda \quad \delta(4)=124
$$

Determine $\delta^{4}(\sigma)$ for $\sigma=1,2,3,4: \delta^{4}(1) \neq \Lambda, \delta^{4}(2)=\Lambda, \delta^{4}(3)=\Lambda, \delta^{4}(4) \neq \Lambda$. 
So $\tilde{\Sigma}=\{1,4\}$, and $\tilde{Q}=\langle\{1,4\}, \tilde{\delta}, 441\rangle$ with

$$
\tilde{\delta}(1)=4 \quad \tilde{\delta}(4)=14 \text {. }
$$

Remark: In larger D0L-systems, the computation of $\delta^{n}(\sigma)$ for all $\sigma \in \Sigma$ can be tedious. An easier way of obtaining $\tilde{G}$ is the construction of intermediate reduced systems. By omitting from $\Sigma$ those $\sigma$ for which $\delta(\sigma)=\Lambda$, one obtains $\Sigma^{\prime}$ and the corresponding $G^{\prime}=\left\langle\Sigma^{\prime}, \delta^{\prime}, w_{0}^{\prime}\right\rangle$. From $G^{\prime}, G^{\prime \prime}$ is constructed, and so on until $G^{(k+1)}=G^{(k)}$ for some $k . Q^{(k)}$ is then the desired $\tilde{G}$.

I omit the proof that the method is equivalent to the "direct" one. In the example, the procedure goes on as follows:

$$
\begin{aligned}
& G^{\prime}=\left\langle\{1,2,4\}, \delta^{\prime}, 4421\right\rangle \text { with } \delta^{\prime}(1)=42, \delta^{\prime}(2)=\Lambda, \delta^{\prime}(4)=124 \\
& G^{\prime \prime}=\left\langle\{1,4\}, \delta^{\prime \prime}, 441\right\rangle \quad \text { with } \delta^{\prime \prime}(1)=4, \delta^{\prime \prime}(4)=14 \\
& G^{\prime \prime}=G^{\prime \prime}, \text { so } \tilde{G}=G^{\prime \prime} .
\end{aligned}
$$

The construction of $\tilde{G}$, the propagating "backbone" of $G$, is essential in the decision procedure for the membership question " $w \in L(G)$ ?" (it still must be proved, of course, that $\tilde{G}$ is propagating). The procedure is, roughly speaking, the following: it is first determined whether $\tilde{w} \in L(\tilde{G})$. One cannot find a positive answer to that question without obtaining a production tree, and that tree is used to decide whether $w \in L(G)$. As I shall show, the procedure is not uniform, but depends on an intermediate result, namely, the finiteness of $L(\tilde{G})$.

Lemma 4. $\tilde{G}$ is propagating.

Proof: Let $\sigma \in \widetilde{\Sigma}$. By construction of $\tilde{G}, \delta^{n}(\sigma)$ is not empty. Consider an arbitrary letter, say $\sigma^{\prime}$, in the word $\delta^{n}(\sigma)$; this word can then be written as $w^{\prime} \sigma^{\prime} w^{\prime \prime}$ for some $w^{\prime}, w^{\prime \prime} \in \Sigma^{*}$. Consider the line of predecessors of $\sigma^{\prime}$ : $\sigma=\tau_{0}, \tau_{1}, \ldots, \tau_{n-1}, \tau_{n}=\sigma^{\prime}$. This line is unique, and $\delta\left(\tau_{i}\right)=a_{i+1} \tau_{i+1} b_{i+1}$ (for some $a_{i+1}$ and $b_{i+1} \in \Sigma^{*}$ ) for each $i$.

As $\# \Sigma=n$, at least two of these $\tau$ 's are equal, say $\tau_{k+j}=\tau_{k}$. Then also $\tau_{n}=\tau_{n-j}$. Hence $\delta\left(\tau_{n}\right)=\delta\left(\tau_{n-1}\right) \neq \Lambda$.

Now

$$
\delta^{n} \circ \delta\left(\tau_{0}\right)=\delta \circ \delta^{n}\left(\tau_{0}\right)=\delta\left(w^{\prime} \tau_{n} w^{\prime \prime}\right)=\delta\left(w^{\prime}\right) \delta\left(\tau_{n}\right) \delta\left(w^{\prime \prime}\right) \neq \Lambda,
$$

so $\chi \circ \delta(\sigma) \neq \Lambda$; in other words, $\tilde{\delta}(\sigma) \neq \Lambda$.

This holds for all $\sigma \in \widetilde{\Sigma}$; so $\widetilde{G}$ is propagating.

LEMMA 5. $\tilde{\delta}(\tilde{w})=\widetilde{\delta(w)}$.

Proof: Let $w=\sigma_{1} \ldots \sigma_{m}$.

$$
\begin{aligned}
\tilde{\delta}(\tilde{w}) & =\chi \circ \delta\left(\chi\left(\sigma_{1}\right) \ldots \chi\left(\sigma_{m}\right)\right) \\
& =\chi \circ \delta \circ \chi\left(\sigma_{1}\right) \ldots \chi \circ \delta \circ \chi\left(\sigma_{m}\right) .
\end{aligned}
$$

4 Indagationes 
If $\sigma_{i} \in \tilde{\Sigma}$, then $\chi \circ \delta \circ \chi\left(\sigma_{i}\right)=\chi \circ \delta\left(\sigma_{i}\right)$.

If $\sigma_{i} \notin \widetilde{\Sigma}$, then $\chi\left(\sigma_{i}\right)=\Lambda \Rightarrow \delta^{n}\left(\sigma_{i}\right)=\Lambda \Rightarrow \delta^{n} \circ \delta\left(\sigma_{i}\right)=\Lambda \Rightarrow \chi \circ \delta\left(\sigma_{i}\right)=\Lambda$. On the other hand, $\chi \circ \delta \circ \chi\left(\sigma_{i}\right)=\Lambda$.

So, in both cases, $\chi \circ \delta \circ \chi\left(\sigma_{i}\right)=\chi \circ \delta\left(\sigma_{t}\right)$.

Hence $\tilde{\delta}(\tilde{w})=\chi \circ \delta\left(\sigma_{1}\right) \ldots \chi \circ \delta\left(\sigma_{m}\right)=\chi \circ \delta(w)=\widetilde{\delta(w)}$.

Coroluary: If $\widetilde{L(G)}$ is defined as $\{\tilde{w} \mid w \in L(G)\}$, then $L(\widetilde{G})=\widetilde{L(G)}$.

Lamma 6. Let $G=\left\langle\Sigma, \delta, w_{0}\right\rangle$ be a DoL-system, and $L=L(G)$. Then $L$ is finite if and only if $\tilde{L}$ is finite.

Proof : $\Rightarrow$ : trivial.

$\Leftarrow:$ Let $\# \Sigma=N ; \max _{\sigma \in \Sigma}|\delta(\sigma)|=K ; \max _{w \in \tilde{L}}|w|=M$.

Vital and mortal letters are letters from $\tilde{\Sigma}$ and $\Sigma-\tilde{\Sigma}$, respectively.

In each word of $L$, every mortal letter stems from a first mortal predecessor (FMP), which is unique.

Since $G$ is determinate, the words of $L$ are produced in a fixed order, so $w_{q}\left(=\delta^{q}\left(w_{0}\right)\right)$ refers to only one word.

If $\sigma$ is mortal, then, by definition,

$$
\delta^{N}(\sigma)=\Lambda
$$

It follows from (i) that a word stemming from a mortal letter can not be longer than $K^{N-1}$ :

$$
(\forall p)(\forall \sigma \in \Sigma-\tilde{\Sigma})\left(\left|\delta^{p}(\sigma)\right| \leqslant K^{N-1}\right) .
$$

Now consider an arbitrary word $w_{q} \in L$ and an arbitrary mortal letter in it. According to (i), the FMP of this letter can only belong to one of the $N-1$ words preceding $w_{q}$. By its definition, each FMP is the immediate successor of a vital letter. Since a word contains no more than $M$ vital letters, the next word contains no more than $M \cdot K$ FMPs (of letters in $w_{q}$ ). All $w_{q}$ 's FMPs belong to the $N-1$ words preceding $w_{q}$, so the set of $w_{q}$ 's FMPs contains no more than $(N-1) M K$ letters. According to (ii), each FMP can only produce strings of length $K^{N-1}$ or less, therefore the number of mortal letters in $w_{q}$ is certainly not larger than $(N-1) M K \cdot K^{N-1}$.

By addition,

so $L$ is finite.

$$
\left|w_{q}\right| \leqslant(N-1) M K^{N}+M
$$

Now it becomes possible to make a rough estimate of $\# L$ if $L$ is finite. Again, put \# $\#=N$, $\max _{\tilde{w} \in \tilde{L}}|\tilde{w}|=M$, and $\max _{\sigma \in \Sigma}|\delta(\sigma)|=K$.

By lemma 3, \#L $L_{\leqslant} N^{N}+N$.

If one puts $M=\left|\tilde{w}_{0}\right| \cdot K^{N^{N}+N}$, then

$\tilde{w} \leqslant M$ for all $\tilde{w} \in \tilde{L}$. 
By (iii) in the proof of lemma 6,

Hence

$$
\left|w_{q}\right| \leqslant(N-1) M K^{N}+M
$$

$$
\# L \leqslant N^{(N-1) M K^{N}+M} \text {. }
$$

This means that someone writing out a finite $L(G)$ has the advantage of knowing an upper bound to the amount of work he has to do.

Theorem: In D0L-systems the membership question is effectively decidable.

Proof: Let $G=\left\langle\Sigma, \delta, w_{0}\right\rangle$ be a D0L-system, with $\# \Sigma=n$.

Given $w \in \Sigma^{*}$, the problem is: $w \in L(G)$ ?

Construct $\tilde{G}$ as indicated before. By lemma 4, $\widetilde{G}$ is a PD0L-system.

By lemma 1 (corollary), it can be determined whether $\tilde{w} \in \tilde{L}$.

(i) If $\tilde{w} \notin \tilde{L}$, then $w \notin L$ (lemma 5 , corollary).

(ii) If $\tilde{w} \in \tilde{L}$, then together with this answer an $m$ was found for which $\tilde{\delta}^{m}\left(\tilde{w}_{0}\right)=\tilde{w}$.

Now determine whether $\tilde{L}$ is finite, with the procedure given in lemma 3.

- If $\tilde{L}$ is finite, then, by lemma $6, L$ is finite, and the question can be decided by writing out the whole of $L$.

- If $\tilde{L}$ is infinite, determine whether $\delta^{m}\left(w_{0}\right)=w$.

If so, then of course $w \in L$.

If not, then $w \notin L$. For, if there existed a $p \neq m$ for which $\delta^{p}\left(w_{0}\right)=w$, then also $\tilde{\delta}^{p}\left(\tilde{w}_{0}\right)=\tilde{w}$ (lemma 5$)$.

Together with $\tilde{\delta}^{m}\left(\tilde{w}_{0}\right)=\tilde{w}$, this would imply that $\tilde{L}$ was finite (lemma 2). But, by assumption, $\tilde{L}$ is infinite.

The following example shows the decision procedure as well as the reason why a distinction has to be made between finite and infinite $\tilde{L}$.

Example. $G=\langle\{1,2,3,4,5\}, \delta, 3142\rangle$ with

$$
\delta(1)=24 \quad \delta(2)=3 \quad \delta(3)=15 \quad \delta(4)=5 \quad \delta(5)=\Lambda .
$$

Question: is $w=152435 \in L(G)$ ?

Solution: Construct $\tilde{G}$.

$$
\delta^{5}(1) \neq \Lambda, \delta^{5}(2) \neq \Lambda, \delta^{5}(3) \neq \Lambda, \delta^{5}(4)=\Lambda, \delta^{5}(5)=\Lambda ;
$$

so $\tilde{G}=\langle\{1,2,3\}, \tilde{\delta}, 321\rangle$ with $\tilde{\delta}(1)=2, \tilde{\delta}(2)=3, \tilde{\delta}(3)=1$.

$$
\tilde{w} \in \tilde{L}, \text { for } \tilde{\delta}\left(w_{0}\right)=123=\tilde{w} .
$$

$\tilde{L}$ is finite; $\tilde{w}_{0}=312 \underset{\vec{\sigma}}{\vec{\sigma}} 123 \underset{\vec{\sigma}}{\underset{\sigma}{\longrightarrow}} 231 \underset{\tilde{\sigma}}{\vec{f}} 312$ etc. 
Now if $\tilde{L}$ were infinite, it would be sufficient to take the equality $\tilde{\delta}\left(\tilde{w}_{0}\right)=\tilde{w}$ (which established the relation $\tilde{w} \in \tilde{L}$ ) and determine whether the corresponding $\delta\left(w_{0}\right)=w$ also holds. The result is negative, and for an infinite $L$ the conclusion $w \notin L$ would be valid. But here it is not, as is shown by $\delta^{4}\left(w_{0}\right)=152435=w$.

\section{CoNCLUSTON}

While reading the manuscript of this paper, dr. G. Rozenberg put forward the conjecture that a DoL-language could be accepted by a linear bounded automaton. This proved to be right for D0L-languages and also for the larger class of 0L-languages. As a consequence, 0Llanguages are context-sensitive, and the main theorem of this paper is thereby reduced to a corollary of the new result [5]. Still, this paper gives a direct decision procedure which is unusual in not being uniform, and the reduction of a DoL-system to a PD0L-system may also be of some interest.

\section{ACKNOWLEDGEMENT}

I wish to thank Professor A. Lindenmayer and Dr. D. van Dalen for their stimulating help and useful criticism.

Filosofisch Instituut

Rijksuniversiteit, Utrecht

\section{REFERENCES}

1. DALEN, D. VAN, A note on some gystems of Lindenmayer Mathematical Systems Theory, 5, 128-140 (1971).

2. Herman, G. T., The computing ability of a developmental model for filamentous organisms, Journal of Theoretical Biology, 25, 421-435 (1969).

3. Lindenmayer, A., Mathematical models for cellular interactions in development, I, II, Journal of Theoretical Biology, 18, 280-315 (1968).

4. - Developmental systems without cellular interactions, their languages and grammars J. Theor. Biol. (1971) 30, 455-484 (1970).

5. Rozenberk, G. and P. G. Dodowt, On 0L-languages. Information and Control (in press) (1971). 